\title{
Kadison's antilattice theorem for a synaptic algebra
}

https://doi.org/10.1515/dema-2018-0002

Received September 18, 2017; accepted January 19, 2018

\begin{abstract}
We prove that if $A$ is a synaptic algebra and the orthomodular lattice $P$ of projections in $A$ is complete, then $A$ is a factor if and only if $A$ is an antilattice. We also generalize several other results of R. Kadison pertaining to infima and suprema in operator algebras.
\end{abstract}

Keywords: synaptic algebra, order unit space, Jordan algebra, spectral resolution, antilattice, factor

MSC: Primary 46B40; Secondary 46L89

\section{Introduction}

A synaptic algebra [1-13] is a generalization of the self-adjoint part of several structures based on operator algebras. For instance, although a synaptic algebra $A$ need not be norm complete (i.e., Banach), $A$ is isomorphic to a JC-algebra if and only if it is Banach [6, Theorem 5.3]. Numerous additional examples of synaptic algebras can be found in the references cited above.

In [14], Richard Kadison calls the self-adjoint part $\mathfrak{S}$ of an operator algebra an antilattice if and only if, whenever two elements of $\mathfrak{S}$ have an infimum in $\mathfrak{S}$, then the elements are comparable (i.e., one is less than or equal to the other). As Kadison remarks, "A moments thought shows that this is as strongly nonlattice as a partially ordered vector space can be" [14, p. 505]. He shows that, in important cases, the condition that $\mathfrak{S}$ is an antilattice is equivalent to the condition that the operator algebra in question is a factor (i.e., its center consists only of scalars). Our main theorem in this paper (Theorem 7) is a version of Kadison's result for a synaptic algebra in which the projections form a complete lattice.

\section{Some properties of a synaptic algebra}

Axioms for a synaptic algebra can be found in $[1, \S 1]$ and will not be repeated here. Rather, we shall briefly sketch some of the important features of a synaptic algebra that we shall need below. Readers who have seen operator algebras will be familiar with many of these features-details can be found in the references given in Section 1, especially in [1]. We use 'iff' as an abbreviation for 'if and only if,' the notation := means 'equals by definition,' and $\mathbb{R}$ is the ordered field of real numbers.

In what follows, we assume that $A$ is a synaptic algebra [1, Definition 1.1]. Thus, associated with $A$ is a real or complex associative unital algebra $R$ with unit 1 called the enveloping algebra of $A$, such that, (1) $1 \in A$, (2) $A$ is a real linear subspace of $R$, (3) the linear space $A$ is a partially ordered order-unit normed space with order

David J. Foulis: Emeritus Professor, Department of Mathematics and Statistics, University of MassaChusetts, Amherst, MA, Postal Adress:1 Suttn Court, Amherst, MA 01002, USA, E-mail: foulis@math.umass.edu

*Corresponding Author: Sylvia Pulmannová: Mathematical Institute, Slovak Academy of Sciences, Štefánikova 49, SK-814 73 Bratislava, Slovakia,

E-mail: silvia.pulmannova@mat.savba.sk 
unit 1 and positive cone $A^{+}:=\{a \in A: 0 \leq a\}$, and (4) the order-unit norm of $a \in A$ is denoted and defined by $\|a\|:=\inf \{0<\lambda \in \mathbb{R}:-\lambda 1 \leq a \leq \lambda 1\}$ [15, pp. 67-69]. In important examples, the enveloping algebra $R$ is a unital (concrete or abstract) operator algebra with an adjoint mapping $a \mapsto a^{\star}, A$ is the self-adjoint part of $R$, and $A^{+}=\left\{r r^{\star}: r \in R\right\}$. We shall assume that $A$ is nontrivial, i.e., $A \neq\{0\}$. Then $1 \neq 0$, which enables us, as usual, to identify each scalar $\lambda \in \mathbb{R}$ with the element $\lambda 1 \in A$.

Let $M \subseteq A$. Then $M$ is partially ordered under the restriction of the partial order $\leq$ on $A$. If $m, n \in M$, then the infimum (greatest lower bound) and the supremum (least upper bound) of $m$ and $n$ in $M-$ if they exist-are written as $m \wedge_{M} n$ and $m \vee_{M} n$, respectively. An involution on $M$ is a mapping $m \mapsto m^{\prime}$ that is order reversing (i.e., $m \leq n \Rightarrow n^{\prime} \leq m^{\prime}$ ) and of order two (i.e., $\left(m^{\prime}\right)^{\prime}=m$ ). Such an involution provides a "duality" between existing infima and suprema in $M$ according to $m \wedge_{M} n=\left(m^{\prime} \vee_{M} n^{\prime}\right)^{\prime}$ and $m \vee_{M} n=\left(m^{\prime} \wedge_{M} n^{\prime}\right)^{\prime}$. Note that the mapping $a \mapsto-a$ is an involution on $A$ itself.

Let $a, b \in A$. Then the product $a b$ is calculated in $R$ and may or may not belong to $A$; however, it is assumed that $0 \leq a^{2} \in A$. Consequently, the Jordan product

$$
a \odot b:=\frac{1}{2}(a b+b a)=\frac{1}{2}\left((a+b)^{2}-a^{2}-b^{2}\right) \in A,
$$

so $A$ is a real unital special Jordan algebra under $\odot$ [16]. If $a$ commutes with $b$, i.e., $a b=b a$ in $R$, we write $a C b$. Evidently, if $a C b$, then $a b=a \odot b \in A$. It can be shown that $a b=0$ iff $b a=0$. Also, if $a, b \in A^{+}$and $a C b$, then $a b \in A^{+}$. As a consequence, if $a, b, c \in A, a \leq b, 0 \leq c, c C a$, and $c C b$, then $c a \leq c b$. Indeed, by the hypotheses, $0 \leq c, b-a$ and $c C(b-a)$, whence $0 \leq c b-c a$, i.e., $c a \leq c b$.

Suppose that $a, b \in A$ and put $c:=2(a \odot b)$. Then $a b a=a \odot c-a^{2} \odot b \in A$ and the mapping $b \mapsto a b a$ is called the quadratic mapping on $A$ determined by $a$. It can be shown that the quadratic mapping $b \mapsto a b a$ is both linear and order preserving on $A$.

An element $e \in A$, such that $0 \leq e \leq 1$ is called an effect. It can be shown that $e \in A$ is an effect iff $e^{2} \leq e$. The subset $E:=\{e \in A: 0 \leq e \leq 1\}$ of $A$ forms a convex effect algebra [17] under the partially defined binary operation obtained by restriction to $E$ of the addition operation + on $A$. The mapping $e \mapsto e^{\perp}:=1-e$, called the orthosupplementation on $E$, is an involution on $E$.

An idempotent element $p=p^{2}$ in $A$ is called a projection, and the set $P:=\left\{p \in A: p=p^{2}\right\}$ of all projections in $A$ is a subset of $E$. In fact, $P$ is precisely the set of all extreme points of the convex set $E$. Under the restriction to $P$ of the partial order on $A, P$ forms an orthomodular lattice (OML) [1, §5], [18, 19], and the orthocomplementation $p \mapsto p^{\perp}:=1-p$ on the OML $P$ is the restriction to $P$ of the orthosupplementation on $E$. The orthocomplementation mapping is an involution on $P$, whence we have an infimum-supremum duality on the lattice $P$. If $e \in E$ and $p \in P$, it can be shown that $e \leq p \Leftrightarrow e=e p \Leftrightarrow e=p e$ and $p \leq e \Leftrightarrow p=$ $p e \Leftrightarrow p=e p$.

Let $p, q \in P$. Then $p \leq q$ iff $p=p q$ iff $p=q p$. We shall write the infimum and the supremum of $p$ and $q$ in the lattice $P$ as $p \wedge q$ and $p \vee q$, respectively, (without subscripts on $\wedge$ and $\vee$ ). If $p C q$, then $p \wedge q=p q=q p$ and $p \vee q=p+q-p q$. The projections $p$ and $q$ are said to be orthogonal, denoted $p \perp q$, iff $p \leq q^{\perp}$, or equivalently iff $q \leq p^{\perp}$. Note that $p \perp q$ iff $p q=0$ iff $q p=0$ iff $p+q=p \vee q$. In particular, for the orthocomplement $p^{\perp}$ of $p$ in $P$, we have, $p \perp p^{\perp}, p C p^{\perp}, p \wedge p^{\perp}=p p^{\perp}=0$ and $p \vee p^{\perp}=p+p^{\perp}=1$.

If $M \subseteq A$, then $C(M):=\{a \in A: a C m$ for all $m \in M\}$ is called the commutant of $M$ and $C C(M):=C(C(M))$ is called the bicommutant of $M$. Evidently, $C(M)$ is a linear subspace (or a vector subspace) of $A$. The subset $M$ of $A$ is said to be commutative iff $m C n$ for all $m, n \in M$, i.e., iff $M \subseteq C(M)$. If $M$ is commutative, then so is $C C(M)$. If $a \in A$, then $C(a):=C(\{a\})$ and $C C(a):=C C(\{a\})$.

If $a \in A^{+}$, then there exists a unique $a^{1 / 2} \in A^{+}$, called the square root of $a$, such that $\left(a^{1 / 2}\right)^{2}=a$. Moreover, $a^{1 / 2} \in C C(a)$. The absolute value of $a \in A$ is denoted and defined by $|a|:=\left(a^{2}\right)^{1 / 2}$ and the positive and negative parts of $a$ are denoted and defined by $a^{+}:=\frac{1}{2}(|a|+a)$ and $a^{-}:=\frac{1}{2}(|a|-a)$, respectively. Then $0 \leq a^{+}, a^{-} \in C C(a), a=a^{+}-a^{-},|a|=a^{+}+a^{-}$, and $a^{+} a^{-}=0$. It turns out that an element $a \in A$ has an inverse $a^{-1}$ in $A$, such that $a a^{-1}=a^{-1} a=1$ iff there exists $0<\epsilon \in \mathbb{R}$, such that $\epsilon \leq|a|$.

If $a \in A$, there exists a unique projection $a^{0} \in P$, called the carrier of $a$, such that, for all $b \in A, a b=$ $0 \Leftrightarrow a^{0} b=0$. (Some authors would refer to $a^{0}$ as the support of $a$.) It fact $a^{0} \in C C(a), a^{0}$ is the smallest projection $p \in P$, such that $a=a p$, and if $e \in E$, then $e^{0}$ is the smallest projection $p \in P$, such that $e \leq p$. See $[1$, Theorem 2.10] for additional properties of the carrier. 
Lemma 1. (i) If $p, q \in P$, then $p \wedge q=p \wedge_{E} q$ and $p \vee q=p \vee_{E} q$.

(ii) If $p, q \in P$, then $p \wedge q$ is also the infimum of $p$ and $q$ in $A^{+}$.

Proof. (i) We have $p, q, p \wedge q \in P \subseteq E$ and $p \wedge q \leq p$, $q$. Suppose $e \in E$ with $e \leq p$, $q$. Then $e^{0} \leq p, q$, whence $e \leq e^{0} \leq p \wedge q$, and therefore $p \wedge q=p \wedge_{E} q$. By duality, $p \vee q=p \vee_{E} q$.

(ii) Clearly, $p \wedge q \in A^{+}$. Suppose that $a \in A^{+}$and $a \leq p, q$. Then $0 \leq a \leq p \leq 1$, so $a \in E$, and since $p \wedge q$ is also the infimum of $p$ and $q$ in $E$ (Lemma 1), we have $a \leq p \wedge q$. Therefore, $p \wedge q$ is the infimum of $p$ and $q$ in $A^{+}$.

Notice that part (ii) of the above lemma is an analogue of [14, Lemma 2].

In view of Lemma 1, no confusion will result if we use the same notation $e \wedge f$ and $e \vee f$ (without subscripts) for existing infima and suprema of effects as we do for infima and suprema of projections. (Actually, the question of when two effects have an infimum in $E$ is important, but not easy to resolve [21].)

There is a very satisfactory spectral theory for $A$ based on the following notions [1, §8]: Let $a \in A$. The spectral resolution of $a$ is the one-parameter family of projections $\left\{p_{\lambda}: \lambda \in \mathbb{R}\right\}$ given by $\left.p_{\lambda}:=\left((a-\lambda)^{+}\right)^{0}\right)^{\perp} \in$ $C C(a)$ for all $\lambda \in \mathbb{R}$. (Recall that $\lambda$ is identified with $\lambda 1 \in A$.) The spectral lower and upper bounds for $a$ are defined by $L:=\sup \{\lambda \in \mathbb{R}: \lambda \leq a\}$ and $U:=\inf \{\lambda \in \mathbb{R}: a \leq \lambda\}$, respectively. By [20, Theorem 3.1], we have $-\infty<L \leq a \leq U<\infty$ and $\|a\|=\max \{|L|,|U|\}$.

A real number $\rho$ belongs to the resolvent set of $a$ iff there is an open interval $I$ in $\mathbb{R}$, such that $\rho \in I$ and $p_{\lambda}=p_{\rho}$ for all $\lambda \in I$. The spectrum of $a$, in symbols $\operatorname{spec}(a)$, which is defined to be the complement in $\mathbb{R}$ of the resolvent set of $a$, is a nonempty closed and bounded subset of $\mathbb{R}$ with all of the expected properties.

Theorem 1. Let $a \in A$ with $0<a$ and let $\left\{p_{\lambda}: \lambda \in \mathbb{R}\right\}$ be the spectral resolution of $a$. For $\lambda \in \mathbb{R}$, define $q_{\lambda}:=1-p_{\lambda}=\left((a-\lambda)^{+}\right)^{0}$. Then: (i) $q_{\lambda} \in C C(a) \subseteq C(a)$; (ii) If $\lambda<0$, then $q_{\lambda}=1$; (iii) $0<q_{0}=a^{\mathrm{o}}$; (iv) If $0<\lambda<\|a\|$, then $0<q_{\lambda}$ and $\lambda q_{\lambda} \leq q_{\lambda} a=a q_{\lambda} \leq a$; (v) If $\lambda \geq\|a\|$, then $q_{\lambda}=0$.

Proof. Part (i) follows from [1, Theorem 8.4 (i)]. Let $L$ and $U$ be the lower and upper spectral bounds for $a$. Since $0<a$, we have $0 \leq L$, whence if $\lambda<0$, then $\lambda<L$, and (ii) then follows from [1, Theorem 8.4 (vi)]. Since $0<a$, we have $a=a^{+}$, whence $q_{0}=\left((a-0)^{+}\right)^{0}=a^{0} \geq 0$. Also, $a^{0} \neq 0$, else $a=0$, contradicting $0<a$, and we have (iii).

As $0 \leq L \leq U$ and $\|a\|=\max \{|L|,|U|\}$, we have $U=\|a\|$. Suppose $0<\lambda<\|A\|=U$. Then by $[1$, Theorem 8.4 (ii)],

$$
\left(1-q_{\lambda}\right)(a-\lambda) \leq 0 \leq q_{\lambda}(a-\lambda) .
$$

Thus, if $q_{\lambda}=0$, then $a \leq \lambda$, so $-\lambda \leq a \leq \lambda$, whence $\|a\| \leq \lambda$, contradicting $\lambda<\|a\|$. Therefore, $0<q_{\lambda}$, and by (1), we have $\lambda q_{\lambda} \leq q_{\lambda} a=a q_{\lambda}$. Since $\left(1-q_{\lambda}\right) C a$ and $0 \leq 1-q_{\lambda}, a$, it follows that $0 \leq\left(1-q_{\lambda}\right) a$, whence we also have $q_{\lambda} a \leq a$, and therefore (iv) holds. Finally, (v) follows from [1, Theorem 8.4 (v)].

Corollary 1. If $0<a \in A$, then there exists $0<\lambda \in \mathbb{R}$ and $0<p \in P$, such that $\lambda p \leq a$.

Proof. Choose $\lambda \in \mathbb{R}$ with $0<\lambda<\|a\|$, and in Theorem 1 let $p=q_{\lambda}$.

An element $s \in A$, such that $s^{2}=1$ is called a symmetry, and two elements $a, b \in A$ are said to be exchanged by the symmetry $s$ iff $s a s=b$ (or, equivalently, iff $s b s=a$ ) [4]. An element $t \in A$ is called a partial symmetry iff $t^{2}=p \in P$, and $a$ and $b$ are exchanged by $t$ iff $t a t=b$ and $t b t=a$. There is a bijective correspondence $s \leftrightarrow p$ between symmetries $s \in A$ and projections $p \in P$ given by $s=2 p-1$ and $p=\frac{1}{2}(s+1)$.

Lemma 2. Let $s \in A$ be a symmetry, $s \neq-1$, and $\lambda, \mu \in \mathbb{R}$. Then $\lambda s \leq \mu \Rightarrow \lambda \leq \mu$.

Proof. Assume the hypotheses. Suppose $\lambda s \leq \mu$, and let $p=\frac{1}{2}(s+1)$ be the projection corresponding to $s$. Then $s=2 p-1=p-p^{\perp}$, and since $s \neq-1$, we have $0<p$. Then $\lambda p-\lambda p^{\perp} \leq \mu$, so $\lambda p=\left(\lambda p-\lambda p^{\perp}\right) p \leq \mu p$, i.e., $0 \leq(\mu-\lambda) p$. If $\mu-\lambda<0$, then $(\mu-\lambda) p<0$, contradicting $0 \leq(\mu-\lambda) p$, whence $0 \leq \mu-\lambda$, so $\lambda \leq \mu$. 
A subset $S \subseteq A$ is called a sub-synaptic algebra of $A$ iff $S$ is a linear subspace of $A, 1 \in S$, and $S$ is closed under the formation of squares, square roots, carriers, and inverses, i.e., $S$ is a synaptic algebra in its own right. For instance, if $M \subseteq A$, then $C(M)$ is a sub-synaptic algebra of $A$. In particular, if $M$ is a commutative subset of $A$, then $C C(M)$ is a commutative sub-synaptic algebra of $A$.

If $p \in P$, then $p A p:=\{p a p: a \in A\}=\{a \in A: a=p a=a p\}$ is a linear subspace of $A$ that is closed under the formation of squares, square roots, carriers, and inverses. It is a synaptic algebra in its own right with $p$ as its unit element and with $\{p r p: r \in R\}$ as its enveloping algebra. The OML of projections in $p A p$ is the interval $P[0, p]:=\{q \in P: q \leq p\}$. If $t$ is a partial symmetry in $A$ with $t^{2}=p$, then $t$ is a symmetry in $p A p$.

The center of $A$ is the commutative sub-synaptic algebra $C(A)$. If $C(A)=\mathbb{R}$, then $A$ is called a factor. For instance, the self-adjoint part $\mathcal{B}^{s a}(\mathfrak{H})$ of the unital $C^{\star}$-algebra of all bounded linear operators on a Hilbert space $\mathfrak{H}$ is a factor.

Theorem 2. The synaptic algebra $A$ is a factor iff the only projections in $C(A)$ are 0 and 1.

Proof. If $C(A)=\mathbb{R}$, then clearly the only projections in $C(A)$ are 0 and 1 . Conversely, suppose that the only projections in $C(A)$ are 0 and 1 and let $a \in C(A)$. By [1, Theorem 8.10], the spectral resolution $\left\{p_{\lambda}: \lambda \in \mathbb{R}\right\}$ of $a$ is contained in $C(A) \cap P$, whence $p_{\lambda} \in\{0,1\}$ for all $\lambda \in \mathbb{R}$. Therefore, by [1, Theorem 8.4 (iii) and (vii)], there exists $\alpha \in \mathbb{R}$, such that $p_{\lambda}=0$ for $\lambda<\alpha$ and $p_{\lambda}=1$ for $\alpha \leq \lambda$, and it follows that $\operatorname{spec}(a)=\{\alpha\}$. Consequently, by [1, Theorem 8.9], $a=\alpha 1=\alpha$.

\section{Two commuting projections}

Lemma 3. (Cf. [14, Proof of Theorem 1]) Suppose that $V$ is a vector subspace of $A, p, q \in P \cap V, p \wedge q \in V$. Suppose further that there exist $w \in P \cap V$ with $p, q \leq w$ and $p \wedge_{V} q$. Put $p_{1}:=p-p \wedge_{V} q$ and $q_{1}:=q-p \wedge_{V} q$. Then:

(i) $p \wedge_{V} q=p \wedge q \in P \cap V$,

(ii) $p_{1}, q_{1} \in P \cap V$ with $p_{1} \wedge_{V} q_{1}=0$,

(iii) $p_{1} q_{1}=q_{1} p_{1}=0$,

(iv) $p$ commutes with $q$.

Proof. Put $g:=p \wedge q \in P \cap V$ and $g_{V}:=p \wedge_{V} q \in V$. By Lemma 1, the infimum of $p$ and $q$ is in $A^{+}$. Hence,

$$
\text { if } a \in A^{+} \text {and } a \leq p, q \text {, then } a \leq g \text {. }
$$

Since $0 \in V$ and $0 \leq p, q$, it follows that $0 \leq g_{V}$. Hence, by (1) with $a:=g_{V}$, we have $g_{V} \leq g$. Also, $g \in V$ and $g \leq p, q$, so $g \leq g_{V}$. Thus, $g_{V}=g$, and we have (i).

By (i), $g_{V} \in P \cap V$, and since $g_{V} \leq p$, it follows that $p_{1}=p-g_{V} \in P \cap V$. Likewise, $q_{1}=q-g_{V} \in P \cap V$. Evidently, $0 \leq p_{1}, q_{1} \in V$. Suppose that $v \in V$ and $v \leq p_{1}, q_{1}$. Then $v+g_{V} \in V$ with $v+g_{V} \leq p, q$, whence $v+g_{V} \leq g_{V}$. So $v \leq 0$, and we have $p_{1} \wedge_{V} q_{1}=0$. This completes the proof of (ii).

Since $p_{1} \leq p \leq w$ and $q_{1} \leq q \leq w$, we have $p_{1}-w, q_{1}-w \leq 0$, whence $p_{1}+q_{1}-w \leq p_{1}, q_{1}$. Therefore, $p_{1}+q_{1}-w \leq p_{1} \wedge_{V} q_{1}=0$, whence $p_{1} \leq w-q_{1}$. As $w$ and $q_{1}$ are projections and $q_{1} \leq w$, it follows that $w-q_{1}$ is a projection, whereupon $p_{1}=p_{1}\left(w-q_{1}\right)=p_{1} w-p_{1} q_{1}=p_{1}-p_{1} q_{1}$. So $p_{1} q_{1}=0$, and we have (iii).

By (i), we have $p_{1}=p-p \wedge q$. So $p \wedge q=p-p_{1} \leq 1-p_{1}$ and it follows that $(p \wedge q) C p_{1}$. Likewise, $(p \wedge q) C q_{1}$. By (iii), $p_{1} C q_{1}$, and therefore $\left(p_{1}+p \wedge q\right) C\left(q_{1}+p \wedge q\right)$, i.e., $p C q$, and we have (iv).

Theorem 3. (Cf. [14, Theorem 1]) Two projections $p$ and $q$ in A commute iff there exists a vector subspace $V$ of $A$, such that $p, q, p \wedge q \in V$, there is a projection $w \in V \cap P$ with $p, q \leq w$, and the infimum $p \wedge_{V} q$ in $V$ of the two projections exists. 
Proof. By Lemma 3, we only have to prove that if $p$ and $q$ commute, then there is a vector subspace $V$ of $A$ with the indicated properties. It suffices to take $V:=C C(\{p, q\})$, noting that $V$ is commutative, and $a \in V \Rightarrow$ $|a|, a^{0} \in V$. Therefore, by [9, Theorem 5.11] $V$ is a lattice.

Corollary 2. Let $p, q \in P$. Then: (i) If $p \wedge_{A} q$ exists, then $p q=q p$; (ii) If $p \wedge_{A} q=0$, then $p q=q p=0$.

Proof. Part (i) is an obvious consequence of Theorem 3. To prove (ii), assume that $p \wedge_{A} q=0$. Then by Lemma 3 with $V=A$, we have $p=p_{1}$ and $q=q_{1}$, whence $p q=0$ by part (iii) of the lemma.

In the following theorem we generalize part (ii) of Corollary 2 to an arbitrary pair of elements $a, b \in A$. In [14, Corollary 8], Kadison obtains this result and its corollary for the special case of $A$ being the self-adjoint part of a $\mathrm{W}^{\star}$-algebra.

Theorem 4. If $a, b \in A$ and $a \wedge_{A} b=0$, then $a b=b a=0$.

Proof. Assume the hypotheses. We have $0=a \wedge_{A} b \leq a, b$, i.e., $a, b \in A^{+}$. We shall use the notion of the generalized infimum of $a$ and $b$, defined and denoted by $a \sqcap b:=\frac{1}{2}(a+b-|a-b|)$ [9, §4]. By [9, Lemma 4.1 (i)], $a \sqcap b \leq a, b$, whence $a \sqcap b \leq a \wedge_{A} b=0$. Thus, since $a, b \in A^{+}, a b=b a=0$ by [9, Lemma 4.4 (ii)].

Corollary 3. If $a, b \in A, c:=a \wedge_{A} b$ exists, $c C a$, and $c C b$, then $a C b$.

Proof. Assume the hypotheses. Then $0 \leq a-c, b-c$, and if $k \in A$ with $k \leq a-c, b-c$, it follows that $k+c \leq a, b$, whence $k+c \leq c$, so $k \leq 0$. Therefore, $(a-c) \wedge_{A}(b-c)=0$. By Theorem 4, $(a-c)(b-c)=(b-c)(a-c)=0$. Consequently, $a b=c b+a c-c^{2}=b c+c a-c^{2}=b a$.

\section{The antilattice theorem}

In this section we prove our main theorem (Theorem 7 ) giving necessary and sufficient conditions for a synaptic algebra $A$ with a complete projection lattice $P$ to be an antilattice. In particular, Theorem 7 shows that a synaptic algebra with a complete projection lattice is an antilattice iff it is a factor. To begin with, we have the following.

Theorem 5. If $A$ is an antilattice, then $A$ is a factor.

Proof. Suppose that $A$ is an antilattice. It will be sufficient to show that the only projections in $C(A)$ are 0 and 1 (Theorem 2). Let $p \in C(A) \cap P$. We claim that $p \wedge_{A} p^{\perp}$ exists and equals 0 . Obviously, $0 \leq p, p^{\perp}$. Suppose $a \in A$ and $a \leq p, p^{\perp}$. As $p \in C(A)$, it follows that $a$ commutes with both $p$ and $p^{\perp}$, whence $p a \leq p p^{\perp}=0$ and $p^{\perp} a \leq p^{\perp} p=0$, and so $a=\left(p+p^{\perp}\right) a=p a+p^{\perp} a \leq 0$. Therefore, $p \wedge_{A} p^{\perp}=0$. Consequently, if $A$ is an antilattice and $p \in C(A) \cap P$, then either $p \leq p^{\perp}$ or $p^{\perp} \leq p$, i.e., $p=0$, or $p=1$.

Lemma 4. If $P$ is a complete $O M L, A$ is a factor, and $0<p, q \in P$ with $p \perp q$, then there exists a symmetry $t \in A$, such that tpt $\leq q$ or $t q t \leq p$. Moreover, $t \neq \pm 1$.

Proof. Assume the hypotheses. By [4, Lemma 8.5], there is a central projection $h \in C(A) \cap P$ and a symmetry $t \in A$, such that $p h$ and a subprojection of $q$ are exchanged by $t$ and $q(1-h)$ and a subprojection of $p$ are exchanged by $t$. Since $A$ is a factor, the central projection $h$ is either 0 or 1 (Theorem 2), whence $t p t \leq q$ or $t q t \leq p$. If $t= \pm 1$, then $t p t=p$ and $t q t=q$, so $p \leq q$ or $q \leq p$. Since $p \perp q, p=0$ or $q=0$ contradicting $0<p, q$.

Lemma 5. Let $a, b \in A$ with $a \wedge_{A} b=0$ and suppose that $p, q \in P, 0<\lambda, \mu \in \mathbb{R}, \lambda p \leq a$ and $\mu q \leq b$. Then $p \wedge_{A} q=p q=q p=0$. 
Proof. Assume the hypotheses and let $\kappa:=\min \{\lambda, \mu\}$. Suppose that $g \in A$ and $g \leq p, q$. Then $\kappa g \leq \kappa p \leq a$ and $\kappa g \leq \kappa q \leq b$, whence $\kappa g \leq 0$. Therefore, $g \leq 0$. Thus, $p \wedge_{A} q=0$, and by Corollary 2 (ii) $p q=q p=0$.

Lemma 6. Suppose that $A$ is not an antilattice. Then there are projections $p, q \in P$ with $p \perp q, 0<p$, $q$, and $p \wedge_{A} q=p q=q p=0$.

Proof. Since $A$ is not an antilattice, there exist $c, d \in A$, such that $c \notin d, d \neq c$, and $c \wedge_{A} d$ exists in $A$. Put $a:=c-c \wedge_{A} d$ and $b:=d-c \wedge_{A} d$. Obviously, $0 \leq a, b$, and since $c \npreceq d$ and $d \notin c$, we have $0<a, b$. Suppose $k \in A$ with $k \leq a, b$. Then $k+c \wedge_{A} d \leq c$, $d$, whence $k+c \wedge_{A} d \leq c \wedge_{A} d$, and it follows that $k \leq 0$. Therefore, $a \wedge_{A} b=0$.

By Corollary 1, there exist projections $0<p, q$ and real numbers $0<\lambda, \mu$, such that $\lambda p \leq a$ and $\mu q \leq b$. Then by Lemma 5, $p \wedge_{A} q=p q=q p=0$, and since $p q=0$, we have $p \perp q$.

Lemma 7. Suppose that $p \in P, p \neq 0,1$, and there exists a symmetry $s \in A$ that exchanges $p$ and $p^{\perp}$. Then there exists $k \in A$ with $k \leq p, p^{\perp}$ but $k \npreceq 0$.

Proof. By the hypotheses, we have $0<p, p^{\perp} \in P$ and there is a symmetry $s \in A$, such that $s p s=p^{\perp}$. Clearly, $s \neq \pm 1$. Put

$$
\alpha:=-\frac{5}{4}, \beta:=-\frac{3}{4}, \gamma:=1 \text {, and } k=2 s-\alpha^{2} \in A .
$$

Note that $k=2 s-\frac{25}{16}, s k s=k$, and

$$
\alpha^{2}-\beta^{2}=1,(\alpha+\beta) \gamma=-2 \text {, and } \beta^{2}+\gamma^{2}=\frac{25}{16} .
$$

We have

$$
s p=p^{\perp} s, p s=s p^{\perp}, s p+p s=s p+s p^{\perp}=s\left(p+p^{\perp}\right)=s 1=s
$$

and

$$
s p^{\perp}+p^{\perp} s=p s+s p=s .
$$

Put $d:=\alpha p+\gamma s+\beta p^{\perp}$. Then by (2) and (3),

$$
\begin{gathered}
0 \leq d^{2}=\alpha^{2} p+\alpha \gamma(p s+s p)+\gamma^{2}+\beta \gamma\left(s p^{\perp}+p^{\perp} s\right)+\beta^{2} p^{\perp} \\
=\alpha^{2} p+\beta^{2}(1-p)+\alpha \gamma s+\gamma^{2}+\beta \gamma s=\left(\alpha^{2}-\beta^{2}\right) p+(\alpha+\beta) \gamma s+\beta^{2}+\gamma^{2} \\
=p-2 s+\frac{25}{16}=p-k,
\end{gathered}
$$

whence $0 \leq p-k$. So $k \leq p$. Therefore, $0 \leq s(p-k) s=s p s-k=p^{\perp}-k$, so $k \leq p^{\perp}$. But $2 \notin \frac{25}{16}$, whence by Lemma $22 s \notin \frac{25}{16}$ and therefore $k=2 s-\frac{25}{16} \npreceq 0$.

Theorem 6. Suppose that whenever $0<p, q \in P$ with $p \perp q$, there exists a symmetry $t$ in $A$, such that tpt $\leq q$ or tqt $\leq p$. Then $A$ is an antilattice.

Proof. Assume the hypothesis. Aiming for a contradiction, we assume that $A$ is not an antilattice. By Lemma 6, there are projections $0<p, q \in P$ with $p \perp q$ and $p \wedge_{A} q=p q=q p=0$. Thus, by hypothesis, there exists a symmetry $t$ in $A$, such that $t p t \leq q$ or $t q t \leq p$. By relabeling if necessary, we can assume that $t p t \leq q$. Thus, $0<t p t \in P$ and $t p t \leq q \leq p^{\perp}$, so tpt $\perp p$. Therefore, $p(t p t)=(t p t) p=0$ and $p \vee t p t=p+t p t$. If $a \in A$ and $a \leq p$, tpt, then $a \leq p, q$. It follows that $a \leq 0$ and hence $p \wedge_{A} t p t=0$.

Now we are going to drop down to the synaptic algebra $A_{1} \subseteq A$ defined by $A_{1}:=(p+t p t) A(p+t p t)$ with unit element $u:=p+t p t$. The projection lattice of $A_{1}$ is the interval $P[0, u]=\{q \in P: q \leq u\}$ in $P$. We have $0<p$, tpt $\in P[0, u]$ with $p(t p t)=(t p t) p=0$ and $p+t p t=p \vee t p t=u$, whence tpt is the orthocomplement of $p$ in $A_{1}$. Clearly, $p \wedge_{A_{1}}$ tpt $=0$. Put $s:=t p+p t$. Then $s$ is a partial symmetry in $A$ with $s^{2}=p+t p t$, and $s(p+t p t)=(p+p t p) s=s$, so $s$ is a symmetry in $A_{1}$. Moreover, sps $=t p t$. Applying Lemma 7 to the synaptic algebra $A_{1}$, we find that there exists $k \in A_{1}$ with $k \leq p$, tpt but $k \npreceq 0$, contradicting $p \wedge_{A_{1}} t p t=0$. 
Theorem 7. Suppose that the OML P of projections in A is complete. Then the following conditions are equivalent:

(i) $A$ is an antilattice.

(ii) A is a factor.

(iii) If $0<p, q \in P$ with $p \perp q$, then there exists a symmetry $t$ in $A$, such that tpt $\leq q$ or tqt $\leq p$.

Proof. Theorem 5 shows that (i) $\Rightarrow$ (ii), Lemma 4 shows that (ii) $\Rightarrow$ (iii), and Theorem 6 shows that (iii) $\Rightarrow$ (i).

Acknowledgement: The second author was supported by grant APVV-16-0073 and grant VEGA No.2/0069/16.

\section{References}

[1] Foulis D. J., Synaptic algebras, Math. Slovaca, 2010, 60, 631-654

[2] Foulis D. J., Pulmannová S., Projections in a synaptic algebra, Order, 2010, 27, 235-257

[3] Foulis D. .., Pulmannová S., Type-decomposition of a synaptic algebra, Found. Phys., 2013, 43, 948-968

[4] Foulis D. J., Pulmannová S., Symmetries in synaptic algebras, Math. Slovaca, 2014, 64, 751-776

[5] Foulis D. J., Pulmannová S., Commutativity in a synaptic algebra, Math. Slovaca, 2016, 66, 469-482

[6] Foulis D. J., Pulmannová S., Banach synaptic algebras, Internat. J. Theoret. Phys. (in press), DOI: 10.1007/s10773-017-3641-y

[7] Foulis D. J., Jenčová A., Pulmannová S., Two projections in a synaptic algebra, Linear Algebra Appl., 2015, 478, 162-187

[8] Foulis D. J., Jenčová A., Pulmannová S., A projection and an effect in a synaptic algebra, Linear Algebra Appl., 2015, 485, 417-441

[9] Foulis D. J., Jenčová A., Pulmannová S., Vector lattices in synaptic algebras, Math. Slovaca, 2017, 57, 1509-1524

[10] Foulis D. J., Jenčová A., Pulmannová S., States and synaptic algebras, Rep. Math. Phys., 2017, 79, 13-32

[11] Foulis D. J., Jenčová A., Pulmannová S., Every synaptic algebra has the monotone square root property, Positivity, 2017, 21, 919-930

[12] Foulis D. J., Jenčová A., Pulmannová S., A Loomis-Sikorski theorem and functional calculus for a generalized Hermitian algebra, Rep. Math. Phys., 2017, 80, 255-275

[13] Pulmannová S., A note on ideals in synaptic algebras, Math. Slovaca, 2012, 62, 1091-1104

[14] Kadison R. V., Order properties of bounded self-adjoint operators, Proc. Amer. Math. Soc., 1951, 2, 505-510

[15] Alfsen E. M., Compact Convex Sets and Boundary Integrals, 1st ed., Springer-Verlag, New York, 1971

[16] McCrimmon K., A taste of Jordan algebras, 1st ed., Springer-Verlag, New York, 2004

[17] Gudder S., Pulmannová S., Bugajski S., Beltrametti E., Convex and linear effect algebras, Rep. Math. Phys., 1999, 44, 359 379

[18] Beran L., Orthomodular Lattices, An Algebraic Approach, 1st ed., D. Reidel Publishing Company, Dordrecht, 1985

[19] Kalmbach G., Orthomodular Lattices, Academic Press, 1st ed., London, New York, 1983

[20] Foulis D. J., Pulmannová S., Spectral resolution in an order unit space, Rep. Math. Phys., 2008, 62, 323-344

[21] Gheondea A., Gudder S., Jonas P., On the infimum of quantum effects, J. Math. Phys., 2005, 46 